\title{
Monotone iterative technique for impulsive fractional evolution equations
}

Jia $\mathrm{Mu}^{*}$ and Yongxiang Li

* Correspondence: mujia88@163. com

Department of Mathematics, Northwest Normal University, Lanzhou, Gansu 730000, People's Republic of China

\begin{abstract}
In this article, the well-known monotone iterative technique is extended for impulsive fractional evolution equations. Under some monotone conditions and noncompactness measure conditions of the nonlinearity, some existence and uniqueness results are obtained. A generalized Gronwall inequality for fractional differential equation is also used. As an application that illustrates the abstract results, an example is given.
\end{abstract}

2000 MSC: 26A33; 34K30; 34K45.

Keywords: impulsive fractional evolution equations, existence and uniqueness, monotone iterative technique, Gronwall inequality, noncompactness measure

\section{Introduction}

In this article, we use the monotone iterative technique to investigate the existence and uniqueness of mild solutions of the impulsive fractional evolution equation in an ordered Banach space $X$ :

$$
\left\{\begin{array}{l}
D^{\alpha} u(t)+A u(t)=f(t, u(t)), \quad t \in I, t \neq t_{k} \\
\left.\Delta u\right|_{t=t_{k}}=I_{k}\left(u\left(t_{k}\right)\right), \quad k=1,2, \ldots, m, \\
u(0)=x_{0} \in X,
\end{array}\right.
$$

where $D^{\alpha}$ is the Caputo fractional derivative of order $0<\alpha<1, A: D(A) \subset X \rightarrow X$ is a linear closed densely defined operator, $-A$ is the infinitesimal generator of an analytic semigroup of uniformly bounded linear operators $T(t)(t \geq 0), I=[0, T], T>0,0=t_{0}$ $<t_{1}<t_{2}<\ldots<t_{m}<t_{m+1}=T, f: I \times X \rightarrow X$ is continuous, $I_{k}: X \rightarrow X$ is a given continuous function, $\left.\Delta u\right|_{t=t_{k}}=u\left(t_{k}^{+}\right)-u\left(t_{k}^{-}\right)$, where $u\left(t_{k}^{+}\right)$and $u\left(t_{k}^{-}\right)$represent the right and left limits of $u(t)$ at $t=t_{k}$, respectively.

Fractional-order models are found to be more adequate than integer-order models in some real-world problems. Fractional derivatives describe the property of memory and heredity of materials, and it is the major advantage of fractional derivatives compared with integer-order derivatives. Fractional differential equations have recently proved to be valuable tools in the modeling of many phenomena in various fields of science. For instance, fractional calculus concepts have been used in the modeling of neurons [1], viscoelastic materials [2]. Other examples from fractional-order dynamics can be found in [3-7] and the references therein. A strong motivation for investigating the initial value problem (1.1) comes from physics. For example, fractional diffusion equations are

(c) $2011 \mathrm{Mu}$ and Li; licensee Springer. This is an Open Access article distributed under the terms of the Creative Commons Attribution License (http://creativecommons.org/licenses/by/2.0), which permits unrestricted use, distribution, and reproduction in any medium, provided the original work is properly cited. 
abstract partial differential equations that involve fractional derivatives in space and time. The time fractional diffusion equation is obtained from the standard diffusion equation by replacing the first-order time derivative with a fractional derivative of order $\alpha \in(0,1)$, namely

$$
\partial_{t}^{\alpha} u(y, t)=A u(y, t), \quad t \geq 0, y \in R,
$$

where $A$ may be linear fractional partial differential operator. For fractional diffusion equations, we can see [8-10] and the references therein.

It is well known that the method of monotone iterative technique has been proved to be an effective and a flexible mechanism. Du and Lakshmikantham [11] established a monotone iterative method for an initial value problem for ordinary differential equation. Later on, many articles used the monotone iterative technique to establish existence and comparison results for nonlinear problems. For evolution equations of integer order $(\alpha=1), \mathrm{Li}$ [12-16] and Yang [17] used this method, in which positive $C_{0}$-semigroup play an important role.

The theory of impulsive differential equations has an extensive physical background and realistic mathematical model, and hence has been emerging as an important area of investigation in recent years, see [18]. Correspondingly, the existence of solutions of impulsive fractional differential equations has also been studied by some authors, see [19-23]. They used the contraction mapping principle, Krasnoselskii's fixed point theorem, Schauder's fixed point theorem, Leray Schauder alternative.

To the best of the authors' knowledge, no results yet exist for the impulsive fractional evolution equations (1.1) by using the monotone iterative technique. The approach via fractional differential inequalities is clearly better suited as in the case of classical results of differential equations and therefore this article choose to proceed in that setup.

Our contribution in this work is to establish the monotone iterative technique for the impulsive fractional evolution equation (1.1). Inspired by [12-17,24-27], under some monotone conditions and noncompactness measure conditions of nonlinearity $f$, we obtain results on the existence and uniqueness of mild solutions of problem (1.1). A generalized Gronwall inequality for fractional differential equation is also applied. At last, to illustrate our main results, we examine sufficient conditions for the main results to an impulsive fractional partial differential diffusion equation.

\section{Preliminaries}

In this section, we introduce notations, definitions and preliminary facts which are used throughout this article.

Definition 2.1. [4] The Riemann-Liouville fractional integral of order $\alpha>0$ with the lower limit zero, of function $f \in L_{1}\left(\mathbb{R}^{+}\right)$, is defined as

$$
I^{\alpha} f(t)=\frac{1}{\Gamma(\alpha)} \int_{0}^{t}(t-s)^{\alpha-1} f(s) d s,
$$

where $\Gamma(\cdot)$ is the Euler gamma function.

Definition 2.2. [4] The Caputo fractional derivative of order $\alpha>0$ with the lower limit zero, $n-1<\alpha<n$, is defined as 


$$
D^{\alpha} f(t)=\frac{1}{\Gamma(n-\alpha)} \int_{0}^{t}(t-s)^{n-\alpha-1} f^{(n)}(s) d s,
$$

where the function $f(t)$ has absolutely continuous derivatives up to order $n-1$. If 0 $<\alpha<1$, then

$$
D^{\alpha} f(t)=\frac{1}{\Gamma(1-\alpha)} \int_{0}^{t} \frac{f^{\prime}(s)}{(t-s)^{\alpha}} d s .
$$

If $f$ is an abstract function with values in $X$, then the integrals and derivatives which appear in (2.1) and (2.2) are taken in Bochner's sense.

Let $X$ be an ordered Banach space with norm $\|\cdot\|$ and partial order $\leq$, whose positive cone $P=\{y \in X \mid y \geq \theta\}$ ( $\theta$ is the zero element of $X)$ is normal with normal constant $N$. Let $C(I, X)$ be the Banach space of all continuous $X$-value functions on interval $I$ with norm $\|u\|_{C}=\max _{t \in I}\|u(t)\|$. Then, $C(I, X)$ is an ordered Banach space reduced by the positive cone $P_{C}=\{u \in C(I, X) \mid u(t) \geq \theta, t \in I\}$. Let $P C(I, X)=\{u: I$ $\rightarrow X \mid u(t)$ is continuous at $t \neq t_{k}$, left continuous at $t=t_{k}$, and $u\left(t_{k}^{+}\right)$exists, $k=1,2$, ..., $m\}$. Evidently, $P C(I, X)$ is an ordered Banach space with norm $\|u\|_{P C}=\sup _{t \in I} \| u$ $(t) \|$ and the partial order $\leq$ reduced by the positive cone $K_{P C}=\{u \in P C(I, X) \mid u(t) \geq$ $\theta, t \in I\} . K_{P C}$ is also normal with the same normal constant $N$. For $u, v \in P C(I, X), u$ $\leq v \Leftrightarrow u(t) \leq v(t)$ for all $t \in I$. For $v, w \in P C(I, X)$ with $v \leq w$, denote the ordered interval $[v, w]=\{u \in P C(I, X) \mid v \leq u \leq w\}$ in $P C(I, X)$, and $[v(t), w(t)]=\{y \in X \mid v(t)$ $\leq y \leq w(t)\}(t \in I)$ in $X$. Set $C^{\alpha, 0}(I, X)=\left\{u \in C(I, X) \mid D^{\alpha} u\right.$ exists and $D^{\alpha} u \in C(I$, $X)\}$. Let $I^{\prime}>=\Lambda\left\{t_{1}, t_{2}, \ldots, t_{m}\right\}$. By $X_{1}$ we denote the Banach space $D(A)$ with the graph norm $\|\cdot\|_{1}=\|\cdot\|+\|A \cdot\|$. An abstract function $u \in P C(I, X) \cap C^{\alpha, 0}\left(I^{\prime}, X\right) \cap C(I$ ', $X_{1}$ ) is called a solution of (1.1) if $u(t)$ satisfies all the equalities of (1.1). We note that - $A$ is the infinitesimal generator of a uniformly bounded analytic semigroup $T(t)(t \geq$ $0)$. This means there exists $M \geq 1$ such that

$$
\|T(t)\| \leq M, \quad t \geq 0 .
$$

Definition 2.3. If $v_{0} \in P C(I, X) \cap C^{\alpha, 0}\left(I^{\prime}, X\right) \cap C\left(I^{\prime}, X_{1}\right)$ and satisfies inequalities

$$
\left\{\begin{array}{l}
D^{\alpha} v_{0}(t)+A v_{0}(t) \leq f\left(t, v_{0}(t)\right), \quad t \in I, t \neq t_{k} \\
\left.\Delta v_{0}\right|_{t=t_{k}} \leq I_{k}\left(v_{0}\left(t_{k}\right)\right), \quad k=1,2, \ldots, m \\
v_{0}(0) \leq x_{0}
\end{array}\right.
$$

then $v_{0}$ is called a lower solution of problem (1.1); if all inequalities of (2.5) are inverse, we call it an upper solution of problem (1.1).

Lemma 2.4. [28-30]If h satisfies a uniform Hölder condition, with exponent $\beta \in(0$, $1]$, then the unique solution of the linear initial value problem (LIVP)

$$
\left\{\begin{array}{l}
D^{\alpha} u(t)+A u(t)=h(t), \quad t \in I, \\
u(0)=x_{0} \in X
\end{array}\right.
$$

is given by

$$
u(t)=U(t) x_{0}+\int_{0}^{t}(t-s)^{\alpha-1} V(t-s) h(s) d s,
$$


where

$$
\begin{aligned}
& U(t)=\int_{0}^{\infty} \zeta_{\alpha}(\theta) T\left(t^{\alpha} \theta\right) d \theta, \quad V(t)=\alpha \int_{0}^{\infty} \theta \zeta_{\alpha}(\theta) T\left(t^{\alpha} \theta\right) d \theta, \\
& \zeta_{\alpha}(\theta)=\frac{1}{\alpha} \theta^{-1-\frac{1}{\alpha}} \rho_{\alpha}\left(\theta^{-\frac{1}{\alpha}}\right), \\
& \rho_{\alpha}(\theta)=\frac{1}{\pi} \sum_{n=0}^{\infty}(-1)^{n-1} \theta^{-\alpha n-1} \frac{\Gamma(n \alpha+1)}{n !} \sin (n \pi \alpha), \quad \theta \in(0, \infty),
\end{aligned}
$$

$\zeta_{\alpha}(\theta)$ is a probability density function defined on $(0, \infty)$.

Remark 2.5. $[29,31-33] \zeta_{\alpha}(\theta) \geq 0, \theta \in(0, \infty), \int_{0}^{\infty} \zeta_{\alpha}(\theta) d \theta=1, \int_{0}^{\infty} \theta \zeta_{\alpha}(\theta) d \theta=\frac{1}{\Gamma(1+\alpha)}$.

Definition 2.6. By the mild solution of IVP (2.6), we mean that the function $u \in C$ $(I, X)$ satisfying the integral equation

$$
u(t)=U(t) x_{0}+\int_{0}^{t}(t-s)^{\alpha-1} V(t-s) h(s) d s,
$$

where $U(t)$ and $V(t)$ are given by (2.8).

Form Definition 2.6, we can easily obtain the following result.

Lemma 2.7. For any $h \in P C(I, X), y_{k} \in X, k=1,2, \ldots, m$, the LIVP

$$
\left\{\begin{array}{l}
D^{\alpha} u(t)+A u(t)=h(t), \quad t \in I, \quad t \neq t_{k} \\
\left.\Delta u\right|_{t=t_{k}}=y_{k}, \quad k=1,2, \ldots, m, \\
u(0)=x_{0} \in X
\end{array}\right.
$$

had the unique mild solution $u \in P C(I, X)$ given by

$$
u(t)=\left\{\begin{array}{l}
U(t) x_{0}+\int_{0}^{t}(t-s)^{\alpha-1} V(t-s) h(s) d s, \quad t \in\left[0, t_{1}\right], \\
U(t)\left[u\left(t_{1}\right)+y_{1}\right]+\int_{t_{1}}^{t}(t-s)^{\alpha-1} V(t-s) h(s) d s, \quad t \in\left(t_{1}, t_{2}\right], \\
\vdots \\
U(t)\left[u\left(t_{m}\right)+y_{m}\right]+\int_{t_{m}}^{t}(t-s)^{\alpha-1} V(t-s) h(s) d s, \quad t \in\left(t_{m}, T\right]
\end{array}\right.
$$

where $U(t)$ and $V(t)$ are given by (2.8).

Remark 2.8. We note that $U(t)$ and $V(t)$ do not possess the semigroup properties. The mild solution of (2.10) can be expressed only by using piecewise functions.

Definition 2.9. An operator family $S(t): X \rightarrow X(t \geq 0)$ in $X$ is called to be positive if for any $y \in P$ and $t \geq 0$ such that $S(t) y \geq \theta$.

From Definition 2.9, if $T(t)(t \geq 0)$ is a positive semigroup generated by - $A, h \geq \theta$, $x_{0} \geq \theta$ and $y_{k} \geq \theta, k=1,2, \ldots, m$, then the mild solution $u \in P C(I, X)$ of (2.10) satisfies $u \geq \theta$. For positive semigroups, one can refer to [12-16].

Now, we recall some properties of the measure of noncompactness will be used later. Let $\mu(\cdot)$ denote the Kuratowski measure of noncompactness of the bounded set. For the details of the definition and properties of the measure of noncompactness, see 
[34]. For any $B \subset C(I, X)$ and $t \in I$, set $B(t)=\{u(t) \mid u \in B\}$. If $B$ is bounded in $C(I$, $X)$, then $B(t)$ is bounded in $X$, and $\mu(B(t)) \leq(B)$.

Lemma 2.10. [35] Let $B=\left\{u_{n}\right\} \subset C(I, X)(n=1,2, \ldots)$ be a bounded and countable set. Then, $\mu(B(t))$ is Lebesgue integral on $I$, and

$$
\mu\left(\left\{\int_{I} u_{n}(t) d t \mid n=1,2, \ldots\right\}\right) \leq 2 \int_{I} \mu(B(t)) d t .
$$

In order to prove our results, we also need a generalized Gronwall inequality for fractional differential equation.

Lemma 2.11. [36] Suppose $b \geq 0, \beta>0$ and $a(t)$ is a nonnegative function locally integrable on $0 \leq t<T$ (some $T \leq+\infty$ ), and suppose $u(t)$ is nonnegative and locally integrable on $0 \leq t<T$ with

$$
u(t) \leq a(t)+b \int_{0}^{t}(t-s)^{\beta-1} u(s) d s
$$

on this interval; then

$$
u(t) \leq a(t)+\int_{0}^{t}\left[\sum_{n=1}^{\infty} \frac{(b \Gamma(\beta))^{n}}{\Gamma(n \beta)}(t-s)^{n \beta-1} a(s)\right] d s, \quad 0 \leq t<T .
$$

\section{Main results}

Theorem 3.1. Let $X$ be an ordered Banach space, whose positive cone $P$ is normal with normal constant $N$. Assume that $T(t)(t \geq 0)$ is positive, the Cauchy problem (1.1) has a lower solution $v_{0} \in C(I, X)$ and an upper solution $w_{0} \in C(I, X)$ with $v_{0} \leq w_{0}$, and the following conditions are satisfied:

$\left(H_{1}\right)$ There exists a constant $C \geq 0$ such that

$$
f\left(t, x_{2}\right)-f\left(t, x_{1}\right) \geq-C\left(x_{2}-x_{1}\right)
$$

for any $t \in I$, and $v_{0}(t) \leq x_{1} \leq x_{2} \leq w_{0}(t)$. That is, $f(t, x)+C x$ is increasing in $x$ for $x \in\left[v_{0}(t), w_{0}(t)\right]$.

$\left(H_{2}\right)$ The impulsive function $I_{k}$ satisfies inequality

$$
I_{k}\left(x_{1}\right) \leq I_{k}\left(x_{2}\right), \quad k=1,2, \ldots, m
$$

for any $t \in I$, and $v_{0}(t) \leq x_{1} \leq x_{2} \leq w_{0}(t)$. That is, $I_{k}(x)$ is increasing in $x$ for $x \in$ $\left[v_{0}(t), w_{0}(t)\right]$.

$\left(H_{3}\right)$ There exists a constant $L \geq 0$ such that

$$
\mu\left(\left\{f\left(t, x_{n}\right)\right\}\right) \leq L \mu\left(\left\{x_{n}\right\}\right)
$$

for any $t \in I$, an increasing or decreasing monotonic sequence $\left\{x_{n}\right\} \subset\left[v_{0}(t), w_{0}(t)\right]$.

Then, the Cauchy problem (1.1) has the minimal and maximal mild solutions between $v_{0}$ and $w_{0}$, which can be obtained by a monotone iterative procedure starting from $v_{0}$ and $w_{0}$, respectively. 
Proof. It is easy to see that $-(A+C I)$ generates an analytic semigroup $S(t)=e^{-C t} T$ $(t)$, and $S(t) \quad(t \geq 0)$ is positive. Let $\Phi(t)=\int_{0}^{\infty} \zeta_{\alpha}(\theta) S\left(t^{\alpha} \theta\right) d \theta$, $\Psi(t)=\alpha \int_{0}^{\infty} \theta \zeta_{\alpha}(\theta) S\left(t^{\alpha} \theta\right) d \theta$. By Remark 2.5, $\Phi(t)(t \geq 0)$ and $\Psi(t)(t \geq 0)$ are positive. By (2.4) and Remark 2.5, we have that

$$
\|\Phi(t)\| \leq M, \quad\|\Psi(t)\| \leq \frac{\alpha}{\Gamma(\alpha+1)} M \triangleq M_{1}, \quad t \geq 0
$$

Let $D=\left[v_{0}, w_{0}\right], J_{1}^{\prime}=\left[t_{0}, t_{1}\right]=\left[0, t_{1}\right], J_{k}^{\prime}=\left(t_{k-1}, t_{k}\right], k=2,3, \ldots, m+1$. We define a mapping $Q: D \rightarrow P C(I, X)$ by

$$
Q u(t)=\left\{\begin{array}{l}
\Phi(t) x_{0}+\int_{0}^{t}(t-s)^{\alpha-1} \Psi(t-s)[f(s, u(s))+C u(s)] d s, \quad t \in J_{1}^{\prime}, \\
\Phi(t)\left[u\left(t_{1}\right)+I_{1}\left(u\left(t_{1}\right)\right)\right]+\int_{t_{1}}^{t}(t-s)^{\alpha-1} \Psi(t-s)[f(s, u(s))+C u(s)] d s, \\
t \in J_{2}^{\prime}, \\
\vdots \\
\Phi(t)\left[u\left(t_{m}\right)+I_{m}\left(u\left(t_{m}\right)\right)\right]+\int_{t_{m}}^{t}(t-s)^{\alpha-1} \Psi(t-s)[f(s, u(s))+C u(s)] d s, \\
t \in J_{m+1}^{\prime} .
\end{array}\right.
$$

Clearly, $Q: D \rightarrow P C(I, X)$ is continuous. By Lemma $2.7, u \in D$ is a mild solution of problem (1.1) if and only if

$$
u=Q u
$$

For $u_{1}, u_{2} \in D$ and $u_{1} \leq u_{2}$, from the positivity of operators $\Phi(t)$ and $\Psi(t),\left(H_{1}\right)$, $\left(H_{2}\right)$, we have inequality

$$
Q u_{1} \leq Q u_{2} .
$$

Now, we show that $v_{0} \leq Q v_{0}, Q w_{0} \leq w_{0}$. Let $D^{\alpha} v_{0}(t)+A v_{0}(t)+C v_{0}(t) \triangleq \sigma(t)$. By Definition 2.3, Lemma 2.7, the positivity of operators $\Phi(t)$ and $\Psi(t)$, for $t \in J_{1}^{\prime}$, we have that

$$
\begin{aligned}
v_{0}(t) & =\Phi(t) v_{0}(0)+\int_{0}^{t}(t-s)^{\alpha-1} \Psi(t-s) \sigma(s) d s \\
& \leq \Phi(t) x_{0}+\int_{0}^{t}(t-s)^{\alpha-1} \Psi(t-s)\left[f\left(s, v_{0}(s)\right)+C v_{0}(s)\right] d s .
\end{aligned}
$$

For $t \in J_{2}^{\prime}$, we have that

$$
\begin{aligned}
v_{0}(t) & =\Phi(t)\left[v_{0}\left(t_{1}\right)+\left.\Delta v_{0}\right|_{t=t_{1}}\right]+\int_{t_{1}}^{t}(t-s)^{\alpha-1} \Psi(t-s) \sigma(s) d s \\
& \leq \Phi(t)\left[v_{0}\left(t_{1}\right)+I_{1}\left(v_{0}\left(t_{1}\right)\right)\right]+\int_{t_{1}}^{t}(t-s)^{\alpha-1} \Psi(t-s)\left[f\left(s, v_{0}(s)\right)+C v_{0}(s)\right] d s
\end{aligned}
$$

Continuing such a process interval by interval to $J_{m+1}^{\prime}$, by (3.2), we obtain that $v_{0} \leq$ $Q v_{0}$. 
Similarly, we can show that $Q w_{0} \leq w_{0}$. For $u \in D$, in view of (3.4), then $v_{0} \leq Q v_{0} \leq$ $Q u \leq Q w_{0} \leq w_{0}$. Thus, $Q: D \rightarrow D$ is an increasing monotonic operator. We can now define the sequences

$$
v_{n}=Q v_{n-1}, \quad w_{n}=Q w_{n-1}, \quad n=1,2, \ldots,
$$

and it follows from (3.4) that

$$
v_{0} \leq v_{1} \leq \cdots v_{n} \leq \cdots \leq w_{n} \leq \cdots \leq w_{1} \leq w_{0}
$$

Let $B=\left\{v_{n}\right\}(n=1,2, \ldots)$ and $B_{0}=\left\{v_{n-1}\right\}(n=1,2, \ldots)$. By (3.6) and the normality of the positive cone $P$, then $B$ and $B_{0}$ are bounded. It follows from $B_{0}=B \cup\left\{v_{0}\right\}$ that $\mu$ $(B(t))=\mu\left(B_{0}(t)\right)$ for $t \in I$. Let

$$
\varphi(t)=\mu(B(t))=\mu\left(B_{0}(t)\right), \quad t \in I .
$$

From $\left(H_{3}\right),(3.1),(3.2),(3.5),(3.7)$, Lemma 2.10 and the positivity of operator $\Psi(t)$, for $t \in J_{1}^{\prime}$, we have that

$$
\begin{aligned}
\varphi(t) & =\mu(B(t))=\mu\left(Q B_{0}(t)\right) \\
& =\mu\left(\left\{\int_{0}^{t}(t-s)^{\alpha-1} \Psi(t-s)\left[f\left(s, v_{n-1}(s)\right)+C v_{n-1}(s)\right] d s \mid n=1,2, \ldots\right\}\right) \\
& \leq 2 \int_{0}^{t} \mu\left(\left\{(t-s)^{\alpha-1} \Psi(t-s)\left[f\left(s, v_{n-1}(s)+C v_{n-1}(s)\right] n=1,2, \ldots\right\}\right) d s\right. \\
& \leq 2 M_{1} \int_{0}^{t}(t-s)^{\alpha-1}(L+C) \mu\left(B_{0}(s)\right) d s \\
& =2 M_{1}(L+C) \int_{0}^{t}(t-s)^{\alpha-1} \varphi(s) d s .
\end{aligned}
$$

By (3.8) and Lemma 2.11, we obtain that $\phi(t) \equiv 0$ on $J_{1}^{\prime}$. In particular, $\mu\left(B\left(t_{1}\right)\right)=\mu$ $\left(B_{0}\left(t_{1}\right)\right)=\phi\left(t_{1}\right)=0$. This means that $B\left(t_{1}\right)$ and $\left.B_{0}\left(t_{1}\right)\right)$ are precompact in $X$. Thus, $I_{1}$ $\left(B_{0}\left(t_{1}\right)\right)$ is pre-compact in $X$ and $\mu\left(I_{1}\left(B_{0}\left(t_{1}\right)\right)\right)=0$. For $t \in J_{2}^{\prime}$, using the same argument as above for $t \in J_{1}^{\prime}$,

we have that

$$
\begin{aligned}
\varphi(t)= & \mu(B(t))=\mu\left(Q B_{0}(t)\right) \\
= & \mu\left(\left\{\Phi(t)\left[v_{n-1}\left(t_{1}\right)+I_{1}\left(v_{n-1}\left(t_{1}\right)\right)\right]\right.\right. \\
& \left.\left.+\int_{t_{1}}^{t}(t-s)^{\alpha-1} \Psi(t-s)\left[f\left(s, v_{n-1}(s)\right)+C v_{n-1}(s)\right] d s \mid n=1,2, \ldots\right)\right\} \\
\leq & M\left[\mu\left(B_{0}\left(t_{1}\right)\right)+\mu\left(I_{1}\left(B_{0}\left(t_{1}\right)\right)\right)\right]+2 M_{1}(L+C) \int_{t_{1}}^{t}(t-s)^{\alpha-1} \varphi(s) d s \\
= & 2 M_{1}(L+C) \int_{t_{1}}^{t}(t-s)^{\alpha-1} \varphi(s) d s .
\end{aligned}
$$

By (3.9) and Lemma 2.11, $\phi(t) \equiv 0$ on $J_{2}^{\prime}$. Then, $\mu\left(B_{0}\left(t_{2}\right)\right)=\mu\left(I_{1}\left(B_{0}\left(t_{2}\right)\right)\right)=0$. Continuing such a process interval by interval to $J_{m+1}^{\prime}$, we can prove that $\phi(t) \equiv 0$ on every $J_{k^{\prime}}^{\prime} k=1,2, \ldots, m+1$. This means $\left\{v_{n}(t)\right\}(n=1,2, \ldots)$ is precompact in $X$ for every $t \in$ I. So, $\left\{v_{n}(t)\right\}$ has a convergent subsequence in $X$. In view of (3.6), we can easily prove that $\left\{v_{n}(t)\right\}$ itself is convergent in $X$. That is, there exist $u(t) \in X$ such that $v_{n}(t) \rightarrow u$ $(t)$ as $n \rightarrow \infty$ for every $t \in I$. By (3.2) and (3.5), we have that 


$$
v_{n}(t)= \begin{cases}\Phi(t) x_{0} \\ +\int_{0}^{t}(t-s)^{\alpha-1} \Psi(t-s)\left[f\left(s, v_{n-1}(s)\right)+C v_{n-1}(s)\right] d s, \quad t \in J_{1^{\prime}}^{\prime} \\ \Phi(t)\left[v_{n-1}\left(t_{1}\right)+I_{1}\left(v_{n-1}\left(t_{1}\right)\right)\right] \\ +\int_{t_{1}}^{t}(t-s)^{\alpha-1} \Psi(t-s)\left[f\left(s, v_{n-1}(s)\right)+C v_{n-1}(s)\right] d s, \quad t \in J_{2^{\prime}}^{\prime} \\ \vdots \\ \Phi(t)\left[v_{n-1}\left(t_{m}\right)+I_{m}\left(v_{n-1}\left(t_{m}\right)\right)\right] \\ +\int_{t_{m}}^{t}(t-s)^{\alpha-1} \Psi(t-s)\left[f\left(s, v_{n-1}(s)\right)+C v_{n-1}(s)\right] d s, \quad t \in J_{m+1}^{\prime} .\end{cases}
$$

Let $n \rightarrow \infty$, then by Lebesgue-dominated convergence theorem, we have that

$$
\underline{u}(t)=\left\{\begin{array}{l}
\Phi(t) x_{0}+\int_{0}^{t}(t-s)^{\alpha-1} \Psi(t-s)[f(s, \underline{u}(s))+C \underline{u}(s)] d s, \quad t \in J_{1}^{\prime}, \\
\Phi(t)\left[\underline{u}\left(t_{1}\right)+I_{1}\left(\underline{u}\left(t_{1}\right)\right)\right] \\
+\int_{t_{1}}^{t}(t-s)^{\alpha-1} \Psi(t-s)[f(s, \underline{u}(s))+C \underline{u}(s)] d s, \quad t \in J_{2}^{\prime}, \\
\vdots \\
\Phi(t)\left[\underline{u}\left(t_{m}\right)+I_{m}\left(\underline{u}\left(t_{m}\right)\right)\right] \\
+\int_{t_{m}}^{t}(t-s)^{\alpha-1} \Psi(t-s)[f(s, \underline{u}(s))+C \underline{u}(s)] d s, \quad t \in J_{m+1}^{\prime},
\end{array}\right.
$$

and $\underline{u} \in C(I, X)$. Then, $\underline{u}=Q \underline{u}$. Similarly, we can prove that there exists $\bar{u} \in C(I, X)$ such that $\bar{u}=Q \bar{u}$. By (3.4), if $u \in D$, and $u$ is a fixed point of $Q$, then $v_{1}=Q v_{0} \leq Q u=$ $u \leq Q w_{0}=w_{1}$. By induction, $v_{n} \leq u \leq w_{n}$. By (3.6) and taking the limit as $n \rightarrow \infty$, we conclude that $v_{0} \leq \underline{u} \leq u \leq \bar{u} \leq w_{0}$. That means that $u, \bar{u}$ are the minimal and maximal fixed points of $Q$ on $\left[v_{0}, w_{0}\right]$, respectively. By (3.3), they are the minimal and maximal mild solutions of the Cauchy problem (1.1) on $\left[v_{0}, w_{0}\right]$, respectively.

Remark 3.2. Theorem 3.1 extend [[37], Theorem 2.1]. Even if $X=\mathbb{R}, A=0$ and $I_{k}=$ $0, k=1,2, \ldots, m$, our results are also new.

Corollary 3.3. Let $X$ be an ordered Banach space, whose positive cone $P$ is regular. Assume that $T(t)(t \geq 0)$ is positive, the Cauchy problem (1.1) has a lower solution $v_{0} \in$ $C(I, X)$ and an upper solution $w_{0} \in C(I, X)$ with $v_{0} \leq w_{0},\left(H_{1}\right)$ and $\left(H_{2}\right)$ hold. Then, the Cauchy problem (1.1) has the minimal and maximal mild solutions between $v_{0}$ and $w_{0}$, which can be obtained by a monotone iterative procedure starting from $v_{0}$ and $w_{0}$, respectively.

Proof. Since $\left(H_{1}\right)$ and $\left(H_{2}\right)$ are satisfied, then (3.6) holds. In regular positive cone $P$, any monotonic and ordered-bounded sequence is convergent. For $t \in I$, let $\left\{x_{n}\right\}$ be an increasing or decreasing sequence in $\left[v_{0}(t), w_{0}(t)\right]$. By $\left(H_{1}\right),\left\{f\left(t, x_{n}\right)+C x_{n}\right\}$ is an ordered-monotonic and ordered-bounded sequence in $X$. Then, $\mu\left\{f\left(t, x_{n}\right)+C x_{n}\right\}=\mu$ $\left(\left\{x_{n}\right\}\right)=0$. By the properties of the measure of noncompactness, we have

$$
\mu\left(\left\{f\left(t, x_{n}\right)\right\}\right) \leq \mu\left(\left\{f\left(t, x_{n}\right)+C x_{n}\right\}\right)+C \mu\left(\left\{x_{n}\right\}\right)=0 .
$$

So, $\left(H_{3}\right)$ holds. Then, by the proof of Theorem 3.1, the proof is then complete. $\square$ 
Corollary 3.4. Let $X$ be an ordered and weakly sequentially complete Banach space, whose positive cone $P$ is normal with normal constant $N$. Assume that $T(t)(t \geq 0)$ is positive, the Cauchy problem (1.1) has a lower solution $v_{0} \in C(I, X)$ and an upper solution $w_{0} \in C(I, X)$ with $v_{0} \geq w_{0},\left(H_{1}\right)$ and $\left(H_{2}\right)$ hold. Then, the Cauchy problem (1.1) has the minimal and maximal mild solutions between $v_{0}$ and $w_{0}$, which can be obtained by a monotone iterative procedure starting from $v_{0}$ and $w_{0}$, respectively.

Proof. Since $X$ is an ordered and weakly sequentially complete Banach space, then the assumption $\left(H_{3}\right)$ holds. In fact, by [[38], Theorem 2.2], any monotonic and orderedbounded sequence is precompact. Let $x_{n}$ be an increasing or decreasing sequence. By $\left(H_{1}\right),\left\{f\left(t, x_{n}\right)+C x_{n}\right\}$ is a monotonic and ordered-bounded sequence. Then, by the properties of the measure of noncompactness, we have

$$
\mu\left(\left\{f\left(t, x_{n}\right)\right\}\right) \leq \mu\left(\left\{f\left(t, x_{n}\right)+C x_{n}\right\}\right)+\mu\left(\left\{C x_{n}\right\}\right)=0 .
$$

So, $\left(H_{3}\right)$ holds. By Theorem 3.1, the proof is then complete. $\square$

Theorem 3.5. Let $X$ be an ordered Banach space, whose positive cone $P$ is normal with normal constant $N$. Assume that $T(t)(t \geq 0)$ is positive, the Cauchy problem (1.1) has a lower solution $v_{0} \in C(I, X)$ and an upper solution $w_{0} \in C(I, X)$ with $v_{0} \leq w_{0}$, $\left(H_{1}\right)$ and $\left(H_{2}\right)$ hold, and the following condition is satisfied:

$\left(H_{4}\right)$ There is a constant $S \geq 0$ such that

$$
f\left(t, x_{2}\right)-f\left(t, x_{1}\right) \leq S\left(x_{2}-x_{1}\right)
$$

for any $t \in I, v_{0}(t) \leq x_{1} \leq x_{2} \leq w_{0}(t)$.

Then, the Cauchy problem (1.1) has the unique mild solution between $v_{0}$ and $w_{0}$, which can be obtained by a monotone iterative procedure starting from $v_{0}$ or $w_{0}$.

Proof. We can find that $\left(H_{1}\right),\left(H_{2}\right)$ and $\left(H_{4}\right)$ imply $\left(H_{3}\right)$. In fact, for $t \in I$, let $\left\{x_{n}\right\} \subset$ $\left[v_{0}(t), w_{0}(t)\right]$ be an increasing sequence. For $m, n=1,2, \ldots$ with $m>n$, by $\left(H_{1}\right)$ and $\left(H_{4}\right)$, we have that

$$
\theta \leq f\left(t, x_{m}\right)-f\left(t, x_{n}\right)+C\left(x_{m}-x_{n}\right) \leq(S+C)\left(x_{m}-x_{n}\right) .
$$

By (3.11) and the normality of positive cone $P$, we have

$$
\left\|f\left(t, x_{m}\right)-f\left(t, x_{n}\right)\right\| \leq(N S+N C+C)\left\|x_{m}-x_{n}\right\| .
$$

From (3.12) and the definition of the measure of noncompactness, we have that

$$
\mu\left(\left\{f\left(t, x_{n}\right)\right\}\right) \leq L \mu\left(\left\{x_{n}\right\}\right),
$$

where $L=N S+N C+C$. Hence, $\left(H_{3}\right)$ holds.

Therefore, by Theorem 3.1, the Cauchy problem (1.1) has the minimal mild solution $\underline{u}$ and the maximal mild solution $\bar{u}$ on $D=\left[v_{0}, w_{0}\right]$. In view of the proof of Theorem 3.1, we show that $u=\bar{u}$. For $t \in J_{1}^{\prime}$, by (3.2), (3.3), $\left(H_{4}\right)$ and the positivity of operator $\Psi$ $(t)$, we have that

$$
\begin{aligned}
\theta & \leq \bar{u}(t)-\underline{u}(t)=Q \bar{u}(t)-Q \underline{u}(t) \\
& =\int_{0}^{t}(t-s)^{\alpha-1} \Psi(t-s)[f(s, \bar{u}(s))-f(s, \underline{u}(s))+C(\bar{u}(s)-\underline{u}(s))] d s \\
& \leq \int_{0}^{t}(t-s)^{\alpha-1} \Psi(t-s)(S+C)(\bar{u}(s)-\underline{u}(s)) d s .
\end{aligned}
$$


By (3.1), (3.13) and the normality of the positive cone $P$, we obtain that

$$
\|\bar{u}(t)-\underline{u}(t)\| \leq N M_{1}(S+C) \int_{0}^{t}(t-s)^{\alpha-1}\|\bar{u}(s)-\underline{u}(s)\| d s .
$$

By Lemma 2.11, then $\underline{u}(t) \equiv \bar{u}(t)$ on $J_{1}^{\prime}$. For $t \in J_{2}^{\prime}$, since $I_{1}\left(\bar{u}\left(t_{1}\right)\right)=I_{1}\left(\underline{u}\left(t_{1}\right)\right)$, using the same argument as above for $t \in J_{1}^{\prime}$, we can prove that

$$
\|\bar{u}(t)-\underline{u}(t)\| \leq N M_{1}(S+C) \int_{t_{1}}^{t}(t-s)^{\alpha-1}\|\bar{u}(s)-\underline{u}(s)\| d s .
$$

Again, by Lemma 2.11, we obtain that $\underline{u}(t) \equiv \bar{u}(t)$ on $J_{2}^{\prime}$. Continuing such a process interval up to $J_{m+1}^{\prime}$, we see that $\underline{u}(t) \equiv \bar{u}(t)$ over the whole of $I$. Hence, $\underline{u}=\bar{u}$ is the unique mild solution of the Cauchy problem (1.1) on $\left[v_{0}, w_{0}\right]$. By the proof of Theorem 3.1, we know it can be obtained by a monotone iterative procedure starting from $v_{0}$ or $w_{0}$. $\square$

\section{Examples}

Example 4.1. In order to illustrate our main results, we consider the impulsive fractional partial differential diffusion equation in $X$

$$
\begin{cases}\partial_{t}^{\alpha} u-\nabla^{2} u=g(y, t, u), & (y, t) \in \Omega \times I, t \neq t_{k}, \\ \left.\Delta u\right|_{t=t_{k}}=J_{k}\left(y, u\left(y, t_{k}\right)\right), & k=1,2, \ldots, m, \\ \left.u\right|_{\partial \Omega}=0, & \\ u(y, 0)=\psi(y), & \end{cases}
$$

where $\partial_{t}^{\alpha}$ is the Caputo fractional partial derivative of order $0<\alpha<1, \nabla^{2}$ is the Laplace operator, $I=[0, T], \Omega \subset \mathbb{R}^{N}$ is a bounded domain with a sufficiently smooth boundary $\partial \Omega, g: \bar{\Omega} \times I \times \mathbb{R} \rightarrow \mathbb{R}$ is continuous, $J_{k}: \bar{\Omega} \times \mathbb{R} \rightarrow \mathbb{R}$ is also continuous, $k=$ $1,2, \ldots, m$.

Let $X=L^{2}(\Omega), P=\left\{v \mid v \in L^{2}(\Omega), v(y) \geq 0\right.$ a.e.y $\left.\in \Omega\right\}$. Then, $X$ is a Banach space, and $P$ is a normal cone in $X$. Define the operator $A$ as follows:

$$
D(A)=H^{2}(\Omega) \cap H_{0}^{1}(\Omega), A u=-\nabla^{2} u .
$$

Then, - $A$ generate an analytic semigroup of uniformly bounded analytic semigroup $T(t)(t \geq 0)$ in $X$ (see [29]). $T(t)(t \geq 0)$ is positive (see [15,16,39,40]). Let $u(t)=u(\cdot, t)$, $f(t, u(t))=g(\cdot, t, u(\cdot, t)), I_{k}\left(u\left(t_{k}\right)\right)=J_{k}\left(\cdot, u\left(\cdot, t_{k}\right)\right)$, then the problem (4.1) can be transformed into the following problem:

$$
\left\{\begin{array}{l}
D^{\alpha} u(t)+A u(t)=f(t, u(t)), \quad t \in I, t \neq t_{k} \\
\left.\Delta u\right|_{t=t_{k}}=I_{k}\left(u\left(t_{k}\right)\right), \quad k=1,2, \ldots, m, \\
u(0)=\psi .
\end{array}\right.
$$

Let $\lambda_{1}$ be the first eigenvalue of $A, \psi_{1}$ is the corresponding eigenfunction. Then, $\lambda_{1} \geq$ $0, \psi_{1}(y) \geq 0$. In order to solve the problem (4.1), we also need the following assumptions:

$\left(O_{1}\right) \psi(\gamma) \in H^{2}(\Omega) \cap H_{0}^{1}(\Omega), 0 \leq \psi(y) \leq \psi_{1}(y), g(y, t, 0) \geq 0, g\left(y, t, \psi_{1}(y)\right) \leq \lambda_{1} \psi_{1}(y), J_{k}$ $(y, 0) \geq 0, J_{k}\left(y, \psi_{1}(y)\right) \leq 0, k=1,2, \ldots, m$.

$\left(\mathrm{O}_{2}\right)$ For any $u_{1}$ and $u_{2}$ in any bounded and ordered interval, and $u_{1} \leq u_{2}$, we have inequality 


$$
J_{k}\left(y, u_{1}\left(y, t_{k}\right)\right) \leq J_{k}\left(y, u_{2}\left(y, t_{k}\right)\right), \quad y \in \Omega, k=1,2, \ldots, m .
$$

$\left(\mathrm{O}_{3}\right)$ The partial derivative $g_{u}^{\prime}(y, t, u)$ is continuous on any bounded domain.

Theorem 4.2. If $\mathrm{O}_{1}, \mathrm{O}_{2}$ and $\mathrm{O}_{3}$ are satisfied, then the problem (4.1) has the unique mild solution.

Proof. From Definition 2.3 and $O_{1}$, we obtain that 0 is a lower solution of (4.2), and $\psi_{1}(y)$ is an upper solution of (4.2). Form $O_{2}$ and $O_{3}$, it is easy to verify that $\left(H_{1}\right),\left(H_{2}\right)$ and $\left(H_{4}\right)$ are satisfied. Therefore, by Theorem 3.5, the problem (4.1) has the unique mild solution.

\section{Acknowledgements}

This research was supported by the NNSFs of China (10871160, 11061031) and Project of NWNU-KJCXGC-3-47.

\section{Authors' contributions}

JM carried out the main part of this article. All authors read and approved the final manuscript.

\section{Competing interests}

The authors declare that they have no competing interests.

Received: 24 June 2011 Accepted: 30 November 2011 Published: 30 November 2011

\section{References}

1. Lundstrom, B, Higgs, M, Spain, W, Fairhall, A: Fractional differentiation by neocortical pyramidal neurons. Nat Neurosci. 11, 1335-1342 (2008). doi:10.1038/nn.2212

2. Rossikhin, Y, Shitikova, M: Application of fractional derivatives to the analysis of damped vibrations of viscoelastic single mass system. Acta Mech. 120, 109-125 (1997). doi:10.1007/BF01174319

3. Hilfer, R: Applications of Fractional Calculus in Physics. World Scientific, Singapore (2000)

4. Kilbas, A, Srivastava, H, Trujillo, J: Theory and Applications of Fractional Differential Equations. Elsevier, Amsterdam (2006)

5. Miller, K, Ross, B: An Introduction to the Fractional Calculus and Fractional Differential Equations. Wiley, New York (1993)

6odlubny, I: Fractional Differential Equations. In Math Sci Eng, vol. 198,Academic Press, San Diego (1999)

7. Samko, S, Kilbas, A, Marichev, O: Fractional Integrals and Derivatives: Theory and Applications. Gordon and Breach Science, Switzerland (1993)

8. Baeumer, B, Kurita, S, Meerschaert, M: Inhomogeneous fractional diffusion equations. J Frac Appl Anal. 8, 375-397 (2005)

9. Henderson, J, Ouahab, A: Fractional functional differential inclusions with finite delay. Nonlinear Anal. 70, 2091-2105 (2009). doi:10.1016/j.na.2008.02.111

10. Meerschaert, M, Benson, D, Scheffler, H, Baeumer, B: Stochastic solution of space-time fractional diffusion equations. Phys Rev E. 65, 1103-1106 (2002)

11. Du, S, Lakshmikantham, V: Monotone iterative technique for differential equations in Banach spaces. J Anal Math Anal. 87, 454-459 (1982). doi:10.1016/0022-247X(82)90134-2

12. Li, Y: Existence and uniqueness of positive periodic solutions for abstract semilinear evolution equations. J Syst Sci Math Sci. 25(6),720-728 (2005)

13. Li, Y: Existence of solutions to initial value problems for abstract semilinear evolution equations. Acta Math Sin. 48(6),1089-1094 (2005)

14. Li, Y: Periodic solutions of semilinear evolution equations in Banach spaces. Acta Math Sin. 41(3),629-636 (1998)

15. Li, Y: The global solutions of initial value problems for abstract semilinear evolution equations. Acta Anal Funct Appl. $3(4), 339-347$ (2001)

16. Li, Y: The positive solutions of abstract semilinear evolution equations and their applications. Acta Math Sin. 39(5),666-672 (1996)

17. Yang, $\mathrm{H}$ : Monotone iterative technique for the initial value problems of impulsive evolution equations in ordered Banach spaces. Abstr Appl Anal 2010, 11 (2010). (Article ID 481648)

18. Lakshmikantham, V, Bainov, D, Simeonov, P: Theory of Impulsive Differential Equations. World Scientific, Singapore (1989)

19. Mophou, G: Existence and uniqueness of mild solutions to impulsive fractional differential equations. Nonlinear Anal. 72, 1604-1615 (2010). doi:10.1016/j.na.2009.08.046

20. Rashid, M, Al-Omari, A: Local and global existence of mild solutions for impulsive fractional semilinear integrodifferential equation. Commun Nonlinear Sci Numer Simul. 16, 3493-3503 (2011). doi:10.1016/j.cnsns.2010.12.043

21. Shu, $X$, Lai, $Y$, Chen, $Y$ : The existence of mild solutions for impulsive fractional partial differential equations. Nonlinear Anal. 74, 2003-2011 (2011). doi:10.1016/j.na.2010.11.007

22. Tai, Z, Wang, X: Controllability of fractional-order impulsive neutral functional infinite delay integrodifferential systems in Banach spaces. Appl Math Lett. 22, 1760-1765 (2009). doi:10.1016/j.aml.2009.06.017

23. Zhang, X, Huang, X, Liu, Z: The existence and uniqueness of mild solutions for impulsive fractional equations with nonlocal conditions and infinite delay. Nonlinear Anal Hybrid Syst. 4,775-781 (2010). doi:10.1016/j.nahs.2010.05.007

24. Li, Y, Liu, Z: Monotone iterative technique for addressing impulsive integro-differential equations in Banach spaces. Nonlinear Anal. 66, 83-92 (2007). doi:10.1016/j.na.2005.11.013

25. Yang, $\mathrm{H}$ : Mixed monotone iterative technique for abstract impulsive evolution equations in Banach space. J Inequal Appl 2010, 15 (2010). (Article ID 293410) 
26. Chen, $\mathrm{P}, \mathrm{Li}, \mathrm{Y}$ : Mixed monotone iterative technique for a class of semilinear impulsive evolution equations in Banach spaces. Nonlinear Anal. 74, 3578-3588 (2011). doi:10.1016/j.na.2011.02.041

27. Chen, P: Mixed monotone iterative technique for impulsive periodic boundary value problems in Banach spaces. Bound Value Problem 2011, 13 (2011). (Article ID 421261). doi:10.1186/1687-2770-2011-13

28. El-Borai, M: Some probability densities and fundamental solutions of fractional evolution equations. Chaos Soliton Fract. 14, 433-440 (2002). doi:10.1016/50960-0779(01)00208-9

29. Wang, J, Zhou, Y, Wei, W: A class of fractional delay nonlinear integrodifferential controlled systems in Banach spaces. Commun Nonlinear Sci Numer Simul. 16, 4049-4059 (2011). doi:10.1016/j.cnsns.2011.02.003

30. Zhou, Y, Jiao, F: Nonlocal Cauchy problem for fractional evolution equations. Nonlinear Anal Real World Appl. 11, 4465-4475 (2010). doi:10.1016/j.nonrwa.2010.05.029

31. Wang, J, Zhou, Y: A class of fractional evolution equations and optimal controls. Nonlinear Anal. 12, 262-272 (2011). doi:10.1016/j.nonrwa.2010.06.013

32. Wang, J, Zhou, $\mathrm{Y}, \mathrm{Wei}, \mathrm{W}, \mathrm{Xu}, \mathrm{H}$ : Nonlocal problems for fractional integrodifferential equations via fractional operators and optimal controls. Comput Math Appl. 62, 1427-1441 (2011). doi:10.1016/j.camwa.2011.02.040

33. Zhou, Y, Jiao, F: Existence of mild solutions for fractional neutral evolution equations. Comput Math Appl. 59, 1063-1077 (2010)

34. Deimling, K: Nonlinear Functional Analysis. Springer, Berlin (1985)

35. Heinz, $\mathrm{H}$ : On the behaviour of measure of noncompactness with respect to differentiation and integration of vectorvalued functions. Nonlinear Anal. 7, 1351-1371 (1983). doi:10.1016/0362-546X(83)90006-8

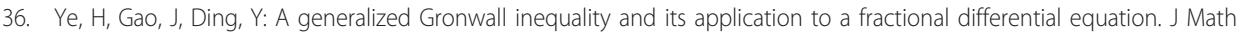
Anal Appl. 328, 1075-1081 (2007). doi:10.1016/j.jmaa.2006.05.061

37. LV, Z, Liang, J, Xiao, T: Solutions to the Cauchy problem for differential equations in Banach spaces with fractional order. Comput Math Appl. 62, 1303-1311 (2011). doi:10.1016/j.camwa.2011.04.027

38. Du, Y: Fixed points of increasing operators in ordered Banach spaces and applications. Appl Anal. 38, 1-20 (1990) doi:10.1080/00036819008839957

39. Liang, J, Liu, J, Xiao, T: Nonlocal problems for integrodifferential equations. Dyn Contin Discrete Impuls Syst Ser (A). 15, 815-824 (2008)

40. Campanto, S: Generation of analytic semigroups by elliptic operators of second order in Hölder space. Ann Sc Norm Sup Pisa Cl Sci. 8, 495-512 (1981)

doi:10.1186/1029-242X-2011-125

Cite this article as: $\mathrm{Mu}$ and Li: Monotone iterative technique for impulsive fractional evolution equations. Journal of Inequalities and Applications 2011 2011:125.

\section{Submit your manuscript to a SpringerOpen ${ }^{\circ}$ journal and benefit from:}

- Convenient online submission

- Rigorous peer review

- Immediate publication on acceptance

- Open access: articles freely available online

- High visibility within the field

- Retaining the copyright to your article

Submit your next manuscript at $\boldsymbol{s p r i n g e r o p e n . c o m ~}$ 